\title{
Effect of COVID 19 Lockdown on a Group of Young Egyptian Athletes
}

\author{
May Nassar ${ }^{1}$, Mohamed Allam ${ }^{1}$, and Mennatallah Shata ${ }^{1}$ \\ ${ }^{1}$ Ain Shams University Faculty of Medicine
}

October 14, 2020

\begin{abstract}
OBJECTIVE: The current study was designed to highlight the physical and psychological health hazards that young Egyptian athletes faced during the COVID-19 lockdown. SETTING: Private sporting club in Cairo. DESIGN: Cross sectional study. PARTICIPANTS: This study included 37 young Egyptian male soccer players whose ages ranged between 9 and 11 years. INTERVENTIONS: Each athlete was subjected to detailed history taking and their anthropometric measurements were taken, mainly weight and height with calculation of their body mass index (BMI). Two questionnaires were filled by each of the enrolled athlete covering their sleep habits and their quality of life (QoL). Finally, the mothers were asked to fill in a questionnaire concerned with depression, anxiety and stress. RESULTS: More than $50 \%$ of the enrolled athletes gained weight during the lockdown. Significantly more young athletes who gained weight reported no compliance to the assigned home exercises and more sleeping hours. The mothers' depression score correlated negatively and their anxiety score correlated positively with the increased BMI of the athletes. The athletes mean QoL Score worsened significantly from March to July 2020. Additionally, a significant negative correlation was found between the increased BMI and the change of QoL. CONCLUSION: The recent pandemic lockdown had both physical and psychological effects on Egyptian young athletes. The increased BMI was significantly reported among those who didn't do home exercises and had a negative correlation with their QoL change throughout the lockdown. Additionally, the mothers' depression and anxiety had a possible reflection on their youngsters' weight gain.
\end{abstract}

Keywords: Athletes; BMI; COVID-19; Depression; Egypt; Lockdown; Soccer; Quality of Life. 'What is already known about this topic?'

The novel coronavirus illness that prevailed late in 2019 (COVID-19) and caused the recent pandemic has had extensive health, social, and economic consequences.

Decision was made to suspend schools for Egyptian children together with curfew restrictions all over the country aiming at alleviating the pandemic repercussions.

Although practicing a physically active lifestyle is recommended to counteract health and mental outcomes of the COVID-19 pandemic, many of the children worldwide ended almost leading a sedentary lifestyle during the lockdown.

\section{'What does this article add?'}

The recent pandemic lockdown had both physical and psychological effects on Egyptian young athletes. The mothers' depression and anxiety had a possible reflection on their youngsters' weight gain.

The increased BMI detected among the young athletes was significantly reported among those who didn't do home exercises and had a negative correlation with their quality of life change throughout the lockdown.

Since athletes' performance is vulnerable to such effects, they should be aware of the need for weight control when outdoors physical activity is necessarily restricted by pandemic control and preventive measures. Com- 
pliance to home exercising schedules with less screen time and video gaming can be of crucial importance to them during these extenuating circumstances. It is prudent to say that following all these previous measures can have a positive effect on the athletes QoL.

\section{INTRODUCTION}

The novel coronavirus illness that prevailed late in 2019 (COVID-19) and caused the recent pandemic has had extensive health, social, and economic consequences. Decision was made to suspend schools for Egyptian children together with curfew restrictions all over the country aiming at alleviating the pandemic repercussions. Although practicing a physically active lifestyle is recommended to counteract health and mental outcomes of the COVID-19 pandemic [1] , many of the children worldwide ended almost leading a sedentary lifestyle during the lockdown. Home confinement had a negative impact on this vulnerable age group and since sporting clubs were closed as well, the athletes among such children faced another hardship.

Sedentary behavior and decreased daily physical activity are associated with copious adverse health effects that collectively predispose to weight gain. Another consequence of lockdown is reduced exposure to daylight and the accompanying disturbance in physical activity patterns, with interruption in meal timing and sleep patterns. Ultimately, circadian rhythms are disrupted, predisposing susceptible individuals to a plethora of metabolic abnormalities [2] . Additionally, psychological effects of quarantine measures play a pivotal role in all these health hazards [3] .

This study was designed to highlight the physical as well as some of the psychological health hazards that young Egyptian athletes might have faced during the early COVID-19 lockdown measures.

\section{METHODOLOGY}

\section{Study population}

This study included 37 young Egyptian male athletes whose ages ranged between 9 and 11 years. They played soccer in private sporting clubs in Cairo. Their regular routine was 5 weekly practices each is two hours long. The single practice was divided into three parts; warming up for 15 minutes which starts by stretching exercises and ends by running down the soccer field outer track then another half an hour muscle strengthening exercises followed by 30 minutes of soccer tactics and lastly 45 minutes playing soccer in the field. The coach encouraged them to stay active and engage in all sports during school day. Unfortunately, schools were closed on the $25^{\text {th }}$ February 2020 and clubs followed in March 2020. The young athletes were instructed to do daily home exercises in the form of attending three weekly online training sessions 120 minutes long each. Nevertheless, 90 minutes daily attendance was considered sufficient since some athletes' families didn't have high speed unlimited internet subscriptions. The young athletes were supposed to substitute the strengthening muscle exercises for actual soccer playing as most of them had restrictions due to lack of adequate spaces at home. According to their response to these instructions the team was further divided into;

The intense exercise group which included those who did the whole 90- or 120-minutes online training daily and performed all the strengthening and stretching exercises. Attending the daily mental speech session which the coach started for psychological support was also required from this group.

The medium exercise group and this comprised those who could achieve at least 90 minutes 2-3 times per week online training, exercises and psychological support sessions.

The mild exercise group who were only able to do at least once per week the 90 minutes online training and couldn't maintain all exercises or psychological support sessions.

The no exercise group contained those who didn't do any neither daily training nor exercises during the lockdown period. This group wasn't compliant to the psychological support session either.

Methodology 
Informed consents were obtained from the mothers who were the primary care givers for the young athletes enrolled in the current study. Each athlete was subjected to detailed history taking laying stress on their compliance during the lock down period and any family illnesses or deaths witnessed. History of their daily sleeping hours, screen time and video gaming were obtained in details. Their anthropometric measurements were taken in July 2020, mainly weight and height with calculation of their body mass index (BMI) and the values were plotted against the growth charts $[4,5]$. The measurements were compared to the ones recorded in March 2020 during their last training session before the COVID 19 lockdown. Two questionnaires were filled by each of the enrolled athlete. The first one covered their sleep habits [6] and the second was concerned with their quality of life (QoL) [7,8]. Finally, the mothers were asked to fill in a questionnaire concerned with depression, anxiety and stress (DASS-21). The mothers were also asked whether their sons experienced any tics during their home lockdown period.

The Children's Sleep Habits Questionnaire (CSHQ) was utilized in the present study. It is a structured interview to assess sleep problems in school children. The questionnaire consists of 33 items covering eight domains or parameters: bedtime resistance, sleep onset delay, sleep duration, sleep-related anxiety problems, sleep-related breathing disorder, as well as excessive daytime somnolence. It was originally developed by [6]

Regarding the QoL the current study applied the multidimensional PedsQL 4.0 questionnaire created by Varni and his associates to measure the essential core domains for pediatric health related quality of life (HRQoL): Physical functioning, emotional functioning, and social functioning, as delineated by the World Health Organization, as well as school functioning $[7,8]$.

The Arabic version of the Depression Anxiety Stress Scale (DASS-21) was used in the current study and it is based on the original English DASS-21 [9] . It is a set of three self-report scales designed to measure the emotional states of depression, anxiety and stress. Each of the three DASS-21 scales contains 7 items, divided into subscales with similar content. The depression scale assesses dysphoria, hopelessness, devaluation of life, self-deprecation, lack of interest / involvement, anhedonia and inertia. The anxiety scale assesses autonomic arousal, skeletal muscle effects, situational anxiety, and subjective experience of anxious affect. The stress scale is sensitive to levels of chronic nonspecific arousal. It assesses difficulty relaxing, nervous arousal, and being easily upset / agitated, irritable / over-reactive and impatient. Scores for depression, anxiety and stress are calculated by summing the scores for the relevant items.

\section{Statistical Analysis}

The collected data were processed and coded before being analyzed using the IBM SPSS program (Statistical Package for Social Sciences) for Windows Version 20.0. Qualitative data are presented using frequencies and related percentages. Quantitative data are presented using means and standard deviations. An independent samples t-test was used to compare the difference in parametric variables between two independent means of two groups. ANOVA was performed to compare quantitative variables among three categories. The chisquared or Fisher's exact test was performed for qualitative variable analysis. The statistical methods were verified, assuming a significance level of $\mathrm{p}<0.05$.

\section{RESULTS}

Our study included 37 male athletes practicing soccer in a private sporting club in Cairo (Egypt). The mean age of participants was 10.8 (Sd 0.46) years old and ranged between 9 and 11 years old. Only 15 (40.5\%) children did not gain weight, based on BMI, during the lockdown from March to July 2020. The mean change in BMI was 0.51 (Sd 0.76) and ranged between -0.6 and 2.3.

Table (1) compares weight, height, BMI, weight percentile, and weight-for-height Z score in March and July 2020. All these variables increased significantly during the mentioned period.

Out of the enrolled athletes $24(64.9 \%)$ didn't perform home exercises during the lockdown while $10(27 \%)$ carried out mild exercises, $2(5.4 \%)$ were moderately exercising and only one $(2.7 \%)$ fulfilled the intense schedule. 
The mean sleep hours per day during the lockdown were 11.1 ( $\mathrm{Sd} 0.8$ ) hours and ranged between 10 and 13 hours. All participants played video games with an average time of $3.2(\mathrm{Sd} 1.3)$ daily hours and ranged between 1 and 6 hours.

Only $10(27 \%)$ of our enrolled athletes had tics during the lockdown, 5 (13.5\%) reported not feeling healthy during the lockdown, and $9(24.3 \%)$ lost a family member during this particular period.

Participants' mothers have a relatively high depression score with mean of 16.4 ( $\mathrm{Sd} 2.6)$ and range between 12 and 21. They have a mean anxiety score of 16.9 (Sd 1.4) and ranged between 14 and 19. After 4 months of lockdown, the athletes' mothers had a mean stress score of 18.2 ( $\mathrm{Sd} \mathrm{1.3)}$ and ranged between 15 and 20.

Table (2) shows correlations between change in BMI and the next possible independent variables; sleeping hours per day, sleep questionnaire, screen time (hours/day) of the athletes as well as the three DASS-21 domains (depression, anxiety and stress) of their mothers. It is noted that increase in BMI was negatively correlated with their mothers' DASS-21 depression score (P value 0.010$)$ and positively correlated with their mothers' DASS-21 stress score (P value 0.026).

Table (3) compares between children with or without weight gain. There were no significant differences among those two groups regarding screen time (hours/day), sleep questionnaire and video games (hours/day). It also compares children with or without weight gain regarding their mothers' DASS-21 depression, DASS21 anxiety, and DASS-21 stress scores and no significant differences were recorded. The only significant differences were the prolonged sleeping hours among the athletes who gained weight ( $\mathrm{P}$ value 0.038) and that more young athletes who gained weight from March till July 2020 reported no compliance to the assigned home exercise schedules ( $\mathrm{P}$ value 0.013 ).

Lastly, the athletes mean QoL Score filled in March 2020 worsened compared with that of July 2020 [82.8 (Sd 4.6) versus 71.1 (Sd 0.72)] with statistically significant difference ( $\mathrm{P}$ value 0.000 ). The mean QoL change was -11 (Sd 4) and ranged between -3 and -18 . Additionally, a significant negative correlation was found between the change of BMI and the change of QoL from March to July 2020 ( $\mathrm{r}=-0.393, \mathrm{P}$ value 0.016).

\section{DISCUSSION}

Based on BMI, more than half of the enrolled athletes (59.5\%) gained weight during the lockdown from March to July 2020. Weight, height, BMI, weight percentile, and weight-for-height Z score increased significantly during that period. Weight gain among athletes can greatly affect their performance. For many athletes across various sport disciplines there is a direct causal connection between success and low body weight [10]

Contrary to the coach instructions, almost two thirds of the enrolled athletes didn't perform any home exercises. With further analysis significantly more of those athletes who were not complaint to the home exercise program gained weight during the pandemic lockdown. This result comes in agreement with Zeigler and associates [11] who reported lack of exercise as a risk factor for weight gain during the COVID pandemic self-quarantine periods.

Lack of exercise and its related overweight problems can gravely impact the immune system of those athletes. It has been mentioned that although moderate-levels of exercise can boost overall immunity [12], intensive and prolonged physical exertion has been linked with an 'open-window' of impaired immunity up to 72 hours after the exercise [13] . Nevertheless, Campbell and Turner, [14] challenged the latter theory and reinforced that it is a misconception to label any form of acute exercise as immunosuppressive, and, instead, they stated that exercise most likely improves immune competency across the lifespan. Another hit to the immune system can come from the extra weight. In addition to metabolic and cardiovascular obesity-related diseases, recent evidence suggested that obesity might affect immune system function [15] . From another perspective obesity was linked to severity of COVID 19 infection among children in Canada [16] and USA [17] . It is thus clear that exercise is essential for boosting the immune system which is greatly needed during pandemics not to mention the benefits of weight control in this domain. 
The mean sleep hours per day during the lockdown were 11.1 which is within the appropriate sleeping hours of this age group as stated by the American Academy of Sleep Medicine [18] . Although sleeping hours were significantly higher among athletes who gained weight and all of them had CHQS above 41, which reflected their disturbed sleep pattern; the higher CHQS among the athletes who gained weight didn't reach statistical significance. The previous findings are supported by the report of Jarrin and associates [19] regarding the relation between disturbed sleep and weight gain.

All participants reported screen time ranging between 4-10 hours/day and played video games with an average time of 3.2 hours daily. Although both screen time and its important element, which is video gaming, were found increased among athletes who gained weight, these results didn't reach statistical significance. Goncalves and collaborators, [20] reported that screen time is related to obesity among children yet video gaming role is still inconclusive with a promising role of exergame play as a tool for weight reduction [21] .

Only $10(27 \%)$ of our enrolled athletes had tics during the lockdown, 5 (13.5\%) reported feeling unhealthy during the lockdown, and $9(24.3 \%)$ lost a family member during this particular period. Although these points represent psychosocial stress and are among the non-chemical stressors affecting childhood obesity $[22]$, this wasn't the case among our studied young athletes.

Although the participants' mothers had a relatively high depression score it correlated negatively with the increased BMI of the athletes. On the other hand, the high anxiety score of mothers correlated positively with the athletes increase in BMI. Although Marshall and associates [23] reported that maternal depression influences children's weight and may increase risk of obesity, Vehmeijer and associates [24] confirmed the current study findings and reported that maternal anxiety was associated with higher children BMI with no associations observed for maternal depression.

The athletes mean QoL Score filled in March 2020 worsened significantly compared to that of July 2020. The previous finding can be easily interpreted in view of a latest publication by Ghosh and collaborators, [25]. The latter authors reported that being quarantined in homes and institutions may impose greater psychological burden on children than the physical sufferings caused by the virus. School closure, lack of outdoor activity, aberrant dietary and sleeping habits are likely to disrupt children's usual lifestyle and can potentially promote monotony, distress, impatience, annoyance and varied neuropsychiatric manifestations. Additionally, in the current study a significant negative correlation was found between the increase in BMI and the change of QoL from March to July 2020. QoL worsening with the increase in BMI is due to the fact that children and adolescents with obesity frequently report an impaired health-related quality of life [26] , which can be defined as 'the impact of health or disease on physical, mental, and social well-being from the patient's point of view' [27] .

In conclusion, the recent COVID 19 pandemic lockdown had both physical and psychological effects on Egyptian young athletes with plausible interrelations correlating them. Additionally, maternal psychological status during these extreme measures has their reflections on the children. Since athletes' performance is vulnerable to such effects, they should be aware of the need for weight control when outdoors physical activity is necessarily restricted by pandemic control and preventive measures. Compliance to home exercising schedules with less screen time and video gaming can be of crucial importance to them during these extenuating circumstances. It is prudent to say that following all these previous measures can have a positive effect on the athletes QoL.

\section{Acknowledgements}

The authors acknowledge the help provided by the mothers and training coaches of the soccer team as well as the efforts of all young athletes during the history taking and other study procedures.

No funding was obtained for this study.

Table (1): Comparison between weight, height, BMI, weight percentile, and weight-for-height $\mathrm{Z}$ score in March and July 2020. 


\begin{tabular}{llll}
\hline Variable & March 2020 & July 2020 & P value \\
\hline Height & $141.3 \pm 5.3$ & $141.5 \pm 5.2$ & $0.000^{*}$ \\
Weight & $36.5 \pm 6.4$ & $37.6 \pm 6.7$ & $0.000^{*}$ \\
BMI & $18.2 \pm 2.3$ & $18.7 \pm 2.3$ & $0.000^{*}$ \\
Weight percentile & $58.6 \pm 26.5$ & $64.8 \pm 22.8$ & $0.002 \vartheta$ \\
Weight-for-Height Z Score & $0.25 \pm 0.92$ & $0.47 \pm 0.77$ & $0.001 \vartheta$ \\
\hline
\end{tabular}

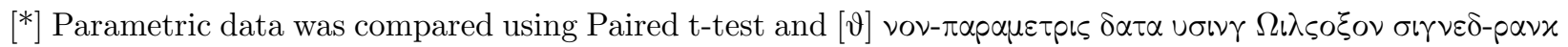
$\tau \varepsilon \sigma \tau$.

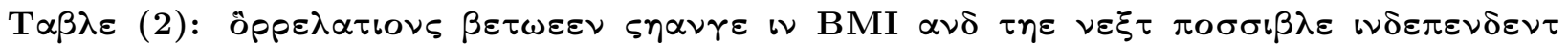

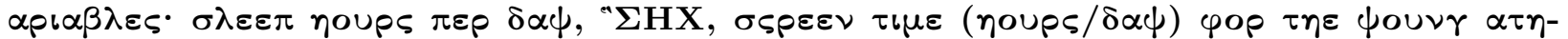

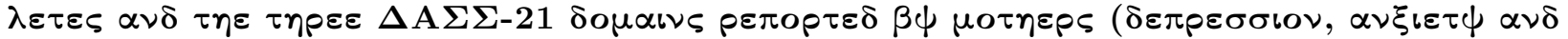
$\sigma \tau \rho \varepsilon \sigma \varsigma)$.

\begin{tabular}{lll}
\hline Change in BMI & Correlation & $\mathrm{P}$ value \\
\hline Sleep hours per day & 0.27 & 0.106 \\
CSHQ & 0.256 & 0.126 \\
Screen time (hours/day) & 0.233 & 0.495 \\
DASS-21 (depression score) & -0.417 & 0.010 \\
DASS-21 (anxiety score) & -0.009 & 0.959 \\
DASS-21 (stress score) & 0.365 & 0.026 \\
\hline
\end{tabular}

Table (3): Comparison between children with or without weight gain regarding sleep hours per day, screen time (hours/day) CSHQ, video games (hours/day) for the young athletes and the three DASS-21 domains reported by mothers (depression, anxiety and stress) as well as home exercise performance, compliance to lockdown and occurrence of tics. ${ }^{1}$

\begin{tabular}{|c|c|c|c|}
\hline Variable & No Weight Gain $(\mathrm{N}=15)$ & Weight Gain $(\mathrm{N}=22)$ & $\mathrm{P}_{\text {value }}{ }^{2}$ \\
\hline Sleep hours per day & $10.7 \pm 0.7$ & $11.3 \pm 0.8$ & 0.038 \\
\hline $\begin{array}{l}\text { Screen time } \\
\text { (hours/day) }\end{array}$ & $6.2 \pm 1.1$ & $7.1 \pm 1.7$ & 0.088 \\
\hline CSHQ & $55.1 \pm 2.9$ & $57.1 \pm 3.3$ & 0.061 \\
\hline $\begin{array}{l}\text { Video games } \\
\text { (hours/day) }\end{array}$ & $3.1 \pm 0.9$ & $3.3 \pm 1.5$ & 0.988 \\
\hline $\begin{array}{l}\text { DASS-21 (depression } \\
\text { score) }\end{array}$ & $17.1 \pm 2.4$ & $16 \pm 2.7$ & 0.196 \\
\hline $\begin{array}{l}\text { DASS-21 (anxiety } \\
\text { score) }\end{array}$ & $17.1 \pm 1.2$ & $16.7 \pm 1.5$ & 0.465 \\
\hline DASS-21 (stress score) & $17.8 \pm 1.0$ & $18.4 \pm 1.4$ & 0.092 \\
\hline $\begin{array}{l}\text { Home exercise Intense } \\
\text { Moderate Mild No }\end{array}$ & $\begin{array}{l}0(0) 2(13.3) 7(46.7) 6 \\
(40)\end{array}$ & $\begin{array}{l}1(4.5) 0(0) 3(13.6) 18 \\
(81.8)\end{array}$ & 0.013 \\
\hline $\begin{array}{l}\text { Compliance to lockdown } \\
\text { Compliant } \\
\text { Non-compliant }\end{array}$ & $10(66.7) 5(33.3)$ & $18(81.8) 4(18.2)$ & 0.438 \\
\hline Tics No Yes & $12(80) 3(20)$ & $15(68.2) 7(31.8)$ & 0.481 \\
\hline
\end{tabular}


${ }^{1}$ Quantitative data are expressed as mean \pm SD; Number in parentheses adjacent to the actual number indicates percentage of cases.

${ }^{2}$ Student's t-test/Mann-Whitney U test for continuous variables and Pearson's Chi square/Fisher's Exact test for categorical variables.

\section{REFERENCES}

1. Jiménez-Pavón D, Carbonell-Baeza A, Lavie CJ. Physical exercise as therapy to fight against the mental and physical consequences of COVID-19 quarantine: special focus in older people. Prog Cardiovasc Dis 2020;63(3):386-8.

2. King AJ, Burke LM, Halson SL, et al. The Challenge of Maintaining Metabolic Health During a Global Pandemic. Sports Med 2020; 50(7):1233-41.

3. Brooks SK, Webster RK, Smith LE, et al. The psychological impact of quarantine and how to reduce it: Rapid review of the evidence. Lancet 2020;395(10227):912-20.

4. Centers for Disease Control and Prevention (CDC): Using the CDC BMI-for-age Growth Charts to Assess Growth in the United States Among Children and Teens Aged 2 Years to 20 Years. https://www.cdc.gov/growthcharts/clinical_charts.htm Page last reviewed: September 9, 2014, accessed August, 2020.

5. WHO: Growth reference. https://www.who.int/growthref/who2007_bmi_for_age/en/ Accessed August, 2020.

6. Owens JA, Spirito A, McGuinn M. The Children's Sleep Habits Questionnaire (CSHQ): psychometric properties of a survey instrument for school-aged children. Sleep 2000; 23:1043-51.

7. Varni JW, Burwinkle TM, Katz ER, et al. The PedsQLin pediatric cancer: reliability and validity of the pediatric quality of life inventory generic core scales, multidimensional fatigue scale, and cancer module. Cancer 2002;94(7):2090-106.

8. Varni JW, Burwinkle TM, Seid M. The PedsQL 4.0 as a school population measure: feasibility, reliability, and validity. Qual Life Res 2006;15:203-15.

9. Lovibond SH and Lovibond PF. Manual for the Depression, Anxiety and Stress Scales, 2nd edn, Sydney, Australia: Psychology Foundation, 1995.

10. Martinsen M, Bratland-Sanda S, Eriksson AK, et al. Dieting to win or to be thin? A study of dieting and disordered eating among adolescent elite athletes and non-athlete controls. Br J Sports Med 2010;1:706.

11. Zeigler Z, Forbes B, Lopez B, et al. Self-quarantine and weight gain related risk factors during the COVID-19 pandemic. Obes Res Clin Pract 2020; 14(3):210-6.

12. Shephard RJ, Shek PN. Potential impact of physical activity and sport on the immune system-a brief review. Br J Sports Med 1994;28(4):247-55.

13. Walsh N.P. Recommendations to maintain immune health in athletes. Eur J Sport Sci 2018;18(6):82031.

14. Campbell JP, Turner JE. Debunking the myth of exercise-induced immune suppression: Redefining the impact of exercise on immunological health across the lifespan. Front Immunol 2018; 9: 648.

15. Umano GR, Pistone C, Tondina E, et al. Pediatric Obesity and the Immune System. Front Pediatr 2019; 7: 487. Published online 2019 Nov 22.

16. Shekerdemian LS, Mahmood NR, Wolfe KK, et al. Characteristics and outcomes of children with coronavirus disease 2019 (COVID-19) infection admitted to US and Canadian pediatric intensive care units. JAMA Pediatr 2020 doi: 10.1001/jamapediatrics.2020.1948.

17. Zachariah P, Johnson CL, Halabi KC, et al. Epidemiology, clinical features, and disease severity in patients with coronavirus disease 2019 (COVID-19) in a children's hospital in New York City, New York. JAMA Pediatr 2020 [Epub ahead of print]

18. Paruthi S, Brooks LJ, D'Ambrosio C, et al. Consensus Statement of the American Academy of Sleep Medicine on the Recommended Amount of Sleep for Healthy Children: Methodology and Discussion. J Clin Sleep Med 2016; 12(11): 1549-61.

19. Jarrin DC, McGarth JJ, Poirier P and QUALITY Cohort Collaborative Group. Autonomic Dysfunc- 
tion: A Possible Pathophysiological Pathway Underlying the Association Between Sleep and Obesity in Children At-Risk for Obesity. J Youth Adolesc 2015; 44(2): 285-97.

20. Goncalves WSF, Byrne R, Viana MT, et al. Parental influences on screen time and weight status among preschool children from Brazil: a cross-sectional study. Int J Behav Nutr Phys Act 2019;16: 27. Published online 2019 Mar 12.

21. Kracht CL, Joseph ED, Staiano AE. Video Games, Obesity, and Children. Curr Obes Rep. Author manuscript; available in PMC 2020 Mar 18.

22. Lichtveld K, Thomas K, Tulve NS. Chemical and non-chemical stressors affecting childhood obesity: a systematic scoping review. J Expo Sci Environ Epidemiol 2018; 28(1):1-12.

23. Marshall SA, Ip EH, Suerken CK, et al. Relationship between maternal depression symptoms and child weight outcomes in Latino farmworkers families. Matern Child Nutr 2018;14(4): e12614.

24. Vehmeijer FOL, Silva CCV, Derks IPM, et al. Associations of Maternal Psychological Distress during Pregnancy with Childhood General and Organ Fat Measures. Childhood Obesity 2019;15(5): 313-22.

25. Ghosh R, Dubey MJ, Chatterjee S, et al. Impact of COVID-19 on children: special focus on the psychosocial aspect. Minerva Pediatr 2020;72:226-35.

26. Schwimmer JB, Burwinkle TM, Varni JW. Health-related quality of life of severely obese children and adolescents. JAMA 2003;289:1813-9.

27. Testa MA, Simonson DC. Assessment of quality of life outcomes. N Engl J Med 1996;334:833-40. 